\title{
Proving Quadratic Derivational Complexities using Context Dependent Interpretations ${ }^{\star}$
}

\author{
Georg Moser and Andreas Schnabl \\ Institute of Computer Science \\ University of Innsbruck, Austria \\ \{georg.moser, andreas.schnabl\}@uibk.ac.at
}

\begin{abstract}
In this paper we study context dependent interpretations, a semantic termination method extending interpretations over the natural numbers, introduced by Hofbauer. We present two subclasses of context dependent interpretations and establish tight upper bounds on the induced derivational complexities. In particular we delineate a class of interpretations that induces quadratic derivational complexity. Furthermore, we present an algorithm for mechanically proving termination of rewrite systems with context dependent interpretations. This algorithm has been implemented and we present ample numerical data for the assessment of the viability of the method.
\end{abstract}

\section{Introduction}

In order to assess the complexity of a (terminating) term rewrite system (TRS for short) it is natural to look at the maximal length of derivation sequences, as suggested by Hofbauer and Lautemann in 11. To be precise, let $\mathcal{R}$ denote a finitely branching and terminating TRS over a finite signature. The derivational complexity function with respect to $\mathcal{R}$ (denoted as $\mathrm{dc}_{\mathcal{R}}$ ) relates the length of the longest derivation sequence to the size of the initial term. For direct termination techniques it is often possible to infer an upper bound on $\mathrm{dc}_{\mathcal{R}}(n)$ from the termination proof of $\mathcal{R}$, cf. [1/2/3/4/5. (Currently it is unknown how to estimate the derivational complexity of a TRS $\mathcal{R}$, if termination of $\mathcal{R}$ has been shown via transformation methods like the dependency pair method or semantic labelling, but see 46] for partial results in this direction.) For example linear derivational complexity can be verified by the use of automata techniques: linear match-bounded TRSs induce linear derivational complexity, see [5]. Unfortunately such a feasible growth rate is not typical. Already termination proofs by polynomial interpretations imply a double-exponential upper bound on the derivational complexity, cf. [1]. In both cases the upper bounds are tight.

However, the tightness of the mentioned bounds does not imply that the upper bounds are always optimal. In particular polynomial interpretations typically overestimate the derivational complexity. In [7] Hofbauer introduced socalled context dependent interpretations as a remedy. These interpretations extend traditional interpretations by introducing an additional parameter. The

\footnotetext{
* This research is supported by FWF (Austrian Science Fund) project P20133.
} 
parameter changes in the course of evaluating a term, which makes the interpretation dependent on the context. The crucial advantage is that context dependent interpretations typically improve the induced bounds on the derivational complexity of TRSs. Furthermore this technique allows the handling of non-simple terminating systems. (See [7] and Section 2 for further details.)

In this paper, we establish theoretical and practical extensions of Hofbauer's approach. As theoretic contributions, we present two subclasses of context dependent interpretations, i.e., we introduce $\Delta$-linear and $\Delta$-restricted interpretations. We show that $\Delta$-linear interpretations induce exponential derivational complexity, while $\Delta$-restricted interpretations induce quadratic derivational complexity. Furthermore, we provide examples showing that these bounds are tight. In [7] it is shown that context dependent interpretations are expressive enough to show termination of TRSs that are not simply terminating. We improve upon this and show that $\Delta$-restricted interpretations suffice here. On the practical side, we design an algorithm that automatically searches for $\Delta$-linear interpretations and $\Delta$-restricted interpretations, which shows that the technique can be mechanised. This answers a question posed by Hofbauer in 7 . The procedure has been implemented and we provide ample numerical data to assess its viability. TRSs with polynomial derivational complexity appear to be of special interest. Thus, we finally compare the applicability of our method to other termination techniques that also induce polynomial derivational complexity.

The remainder of this paper is organised as follows. In the next section we recall basic notions and starting points of this paper. In Section 3 we introduce the class of $\Delta$-linear interpretations and describe the algorithm that mechanises the search for $\Delta$-linear and $\Delta$-restricted interpretations. In Section 4 , we obtain the mentioned results on the derivational complexities induced by either of these interpretations. Furthermore, we show in this section that already $\Delta$-restricted interpretations allow the treatment of non-simple terminating TRSs. Section 5 provides experimental data and finally in Section 6 we conclude and mention future work.

\section{Context Dependent Interpretations}

We assume familiarity with the basics of term rewriting, see [89]. Knowledge of context dependent interpretations [7] will be helpful. Below we recall the basic results from the latter paper in a slightly different, but equivalent way, compare $7[10$. See 7$]$ for the motivation and intuition underlying the introduced concepts.

Let $\mathcal{F}$ be a finite signature, let $\mathcal{V}$ be a set of variables and let $\mathcal{R}$ denote a terminating TRS over $\mathcal{F}$. The induced relation $\rightarrow_{\mathcal{R}}$ is assumed to be finitely branching. We simply write $\rightarrow$ for $\rightarrow_{\mathcal{R}}$ if $\mathcal{R}$ is clear from context. The derivation length of a term $t$ with respect to $\mathcal{R}$ is defined as follows: $\operatorname{dl}_{\mathcal{R}}(t)=\max \{n \mid$ $\left.\exists u t \rightarrow^{n} u\right\}$. The derivational complexity (with respect to $\mathcal{R}$ ) is defined as: $\mathrm{dc}_{\mathcal{R}}(n)=\max \left\{\mathrm{dl}_{\mathcal{R}}(t)|| t \mid \leqslant n\right\}$, where $|t|$ denotes the size of $t$, i.e., the number of symbols of $t$ as usual. (For example the size of the term $\mathrm{f}(\mathrm{a}, x)$ is 3.) We 
say the derivational complexity of $\mathcal{R}$ is linear, quadratic, double-exponential, if $\mathrm{dc}_{\mathcal{R}}(n)$ is bounded by a linear, quadratic, double-exponential function in $n$, respectively. A context dependent $\mathcal{F}$-algebra $(C D A$ for short) $\mathcal{C}$ is a family of $\mathcal{F}$-algebras over the reals parametrised by a set $D \subseteq \mathbb{R}^{+}$of positive reals. A CDA $\mathcal{C}$ associates to each function symbol $f \in \mathcal{F}$ of arity $n$, a collection of $n+1$ mappings: $f_{\mathcal{C}}: D \times\left(\mathbb{R}_{0}^{+}\right)^{n} \rightarrow \mathbb{R}_{0}^{+}$and $f_{\mathcal{C}}^{i}: D \rightarrow D$ for all $1 \leqslant i \leqslant n$. As usual $f_{\mathcal{C}}$ is called interpretation function, while the mappings $f_{\mathcal{C}}^{i}$ are called parameter functions. In addition $\mathcal{C}$ is equipped with a set $\left\{>_{\Delta} \mid \Delta \in D\right\}$ of proper orders, where we define: $z>_{\Delta} z^{\prime}$ if and only if $z-z^{\prime} \geqslant \Delta$.

Let $\mathcal{C}$ be a CDA and let a $\Delta$-assignment denote a mapping: $\alpha: D \times \mathcal{V} \rightarrow \mathbb{R}_{0}^{+}$. We inductively define a mapping $[\alpha, \Delta]_{\mathcal{C}}$ from the set of terms into the set $\mathbb{R}_{0}^{+}$ of non-negative reals:

$$
[\alpha, \Delta]_{\mathcal{C}}(t):= \begin{cases}\alpha(\Delta, t) & \text { if } t \in \mathcal{V} \\ f_{\mathcal{C}}\left(\Delta,\left[\alpha, f_{\mathcal{C}}^{1}(\Delta)\right]_{\mathcal{C}}\left(t_{1}\right), \ldots,\left[\alpha, f_{\mathcal{C}}^{n}(\Delta)\right]_{\mathcal{C}}\left(t_{n}\right)\right) & \text { if } t=f\left(t_{1}, \ldots, t_{n}\right) .\end{cases}
$$

We fix some notational conventions: Due to the special role of the additional variable $\Delta$, we often write $f_{\mathcal{C}}[\Delta]\left(z_{1}, \ldots, z_{n}\right)$ instead of $f_{\mathcal{C}}\left(\Delta, z_{1}, \ldots, z_{n}\right)$. Furthermore, we usually denote the evaluation of $t$ as $[\alpha, \Delta](t)$, if the respective algebra is clear from context.

We say that a CDA $\mathcal{C}$ is $\Delta$-monotone if for all $\Delta \in D$ and for all $a_{1}, \ldots, a_{n}, b \in$ $\mathbb{R}_{0}^{+}$with $a_{i}>_{f_{\mathcal{C}}^{i}(\Delta)} b$ for some $i \in\{1, \ldots, n\}$, we have

$$
f_{\mathcal{C}}[\Delta]\left(a_{1}, \ldots, a_{i}, \ldots, a_{n}\right)>_{\Delta} f_{\mathcal{C}}[\Delta]\left(a_{1}, \ldots, b, \ldots, a_{n}\right) .
$$

Note that if all interpretation functions $f_{\mathcal{C}}[\Delta]$ are weakly monotone with respect to the standard ordering on $\mathbb{R}_{0}^{+}$, then validity of the inequalities

$$
f_{\mathcal{C}}[\Delta]\left(z_{1}, \ldots, z_{i}+f_{\mathcal{C}}^{i}(\Delta), \ldots, z_{n}\right)-f_{\mathcal{C}}[\Delta]\left(z_{1}, \ldots, z_{i}, \ldots, z_{n}\right) \geqslant \Delta,
$$

suffices in order to conclude $\Delta$-monotonicity of $\mathcal{C}$, cf. [7].

A CDA $\mathcal{C}$ is compatible with a TRS $\mathcal{R}$ (or $\mathcal{R}$ is compatible with $\mathcal{C}$ ) if for every rewrite rule $l \rightarrow r \in \mathcal{R}$, every $\Delta \in D$, and any assignment $\alpha:[\alpha, \Delta](l)>_{\Delta}$ $[\alpha, \Delta](r)$ holds.

Example 1 ([7]). As running example, we consider the TRS $\mathcal{R}_{1}$ with the single rewrite rule $\mathrm{a}(\mathrm{b}(x)) \rightarrow \mathrm{b}(\mathrm{a}(x))$. We assume $D=\mathbb{R}^{+}$. The following interpretation and parameter functions

$$
\begin{array}{ll}
\mathrm{a}_{\mathcal{C}}[\Delta](z)=(1+\Delta) z & \mathrm{a}_{\mathcal{C}}^{1}(\Delta)=\frac{\Delta}{1+\Delta} \\
\mathrm{b}_{\mathcal{C}}[\Delta](z)=z+1 & \mathrm{~b}_{\mathcal{C}}^{1}(\Delta)=\Delta,
\end{array}
$$

define a CDA $\mathcal{C}$ that is $\Delta$-monotone and compatible with $\mathcal{R}_{1}$, compare [7].

Theorem 2 ([7]). Let $\mathcal{R}$ be a TRS and suppose that there exists a $\Delta$-monotone and compatible $C D A \mathcal{C}$. Then $\mathcal{R}$ is terminating and

$$
\mathrm{dl}_{\mathcal{R}}(t) \leqslant \inf _{\Delta \in D} \frac{[\alpha, \Delta](t)}{\Delta}
$$

holds for all terms $t \in \mathcal{T}(\mathcal{F}, \mathcal{V})$. 
The next example clarifies the impact of Theorem 2, compare [7.

Example 3. Consider the TRS $\mathcal{R}_{1}$ together with the CDA $\mathcal{C}$ in Example 1 Suppose $c \in \mathcal{F}$ is a constant and $c_{\mathcal{C}}[\Delta]=0$. We assert $D=\mathbb{R}^{+}$. Then we obtain $[\alpha, \Delta]\left(\mathrm{a}^{n}\left(\mathrm{~b}^{m}(\mathrm{c})\right)\right)=(1+\Delta n) m$ and hence:

$$
\inf _{\Delta>0} \frac{[\alpha, \Delta]\left(\mathrm{a}^{n}\left(\mathrm{~b}^{m}(\mathrm{c})\right)\right)}{\Delta}=\inf _{\Delta>0}\left(\frac{1}{\Delta}+n\right) m=n m \geqslant \mathrm{dl}_{\mathcal{R}_{1}}\left(\mathrm{a}^{n}\left(\mathrm{~b}^{m}(\mathrm{c})\right)\right) .
$$

Furthermore, an easy inductive argument reveals: $\mathrm{dl}_{\mathcal{R}_{1}}\left(\mathrm{a}^{n}\left(\mathrm{~b}^{m}(\mathrm{c})\right)\right)=n m$. Hence with respect to the term $a^{n}\left(b^{m}(c)\right)$, compatibility with $\mathcal{C}$ entails an optimal upper bound on the derivation length of $\mathcal{R}_{1}$. This is also true for all ground terms. A proof of $\inf _{\Delta>0} \frac{[\alpha, \Delta](t)}{\Delta}=\mathrm{dl}_{\mathcal{R}_{1}}(t)$ for all $t \in \mathcal{T}(\mathcal{F})$ can be found in [7].

Definition 4. $A \Delta$-quotient is an expression of the form

$$
\frac{\Delta}{a+b \Delta}
$$

where $a, b \in \mathbb{N}$ and either $a>0$ or $b>0 . A \Delta$-quotient $d$ is nontrivial, if $d \neq \Delta$.

Lemma 5. Let $d_{1}, d_{2}$ be $\Delta$-quotients and let $d=d_{1}\left[\Delta:=d_{2}\right]$ denote the result of substituting $d_{2}$ for $\Delta$ in $d_{1}$. Then $d$ is a $\Delta$-quotient.

As usual a polynomial $P$ in the variables $z_{1}, \ldots, z_{n}$ (over the reals) is a finite sum $\sum_{i=1}^{m} c_{i} z_{1}^{i_{1}} \ldots z_{n}^{i_{n}}$. To accommodate $\Delta$-quotients we slightly generalise polynomials.

Definition 6. An extended monomial $M$ in the variables $\Delta$ and $z_{1}, \ldots, z_{n}$ is a finite product $c \cdot \prod_{i} v_{i}$ such that $c$ is an integer and $v_{i}$ is $x^{n}, x \in\left\{\Delta, z_{1}, \ldots, z_{n}\right\}$ or $v_{i}$ is a $\Delta$-quotient. The integer $c$ is called the coefficient and the expression $v_{i}$ a literal. Finally, an extended polynomial $P$ over $\Delta \in D$ and $z_{1}, \ldots, z_{n} \in \mathbb{R}_{0}^{+}$ is a finite sum $\sum_{i} M_{i}$ of extended monomials $M_{i}$ (in $\Delta$ and $z_{1}, \ldots, z_{n}$ ).

Note that the coefficients of an extended polynomial are integers. If the context clarifies what is meant, we will drop the qualifier "extended". Examples 1 and 3 as well as the examples studied in 7 suggest a restricted notion of context dependent algebras. This is the subject of the next definition.

Definition 7. A polynomial context dependent interpretation of $\mathcal{F}$ is a $C D A$ $\left(\mathcal{C},\left\{>_{\Delta} \mid \Delta \in D\right\}\right)$ satisfying the following properties:

- the interpretation function $f_{\mathcal{C}}$ is an extended polynomial,

- the parameter set $D$ equals $\mathbb{R}^{+}$, and

- for each $f \in \mathcal{F}$ the parameter functions $f_{\mathcal{C}}^{i}$ are $\Delta$-quotients.

Lemma 8. Let $\mathcal{C}$ denote a polynomial context dependent interpretation, let $\alpha$ be a $\Delta$-assignment, and let $t$ be a term. Then $[\alpha, \Delta](t)$ is an extended polynomial.

Proof. The lemma is a direct consequence of the definitions and Lemma 5 .

Remark 9. Hofbauer showed in [7] that for any monotone polynomial interpretation compatible with a TRS $\mathcal{R}$, there exists a polynomial context dependent interpretation which is $\Delta$-monotone and compatible with $\mathcal{R}$ and induces at least the same upper bound on the derivational complexity as the polynomial interpretation. 


\section{Automated Search for Context Dependent Interpretations}

One approach to find context dependent interpretations (semi-)automatically was already mentioned in Hofbauer's paper 7 . A given polynomial interpretation is suitably lifted to a context dependent interpretation such that monotonicity and compatibility are preserved, but the upper bound on the derivational complexity is often improved. Unfortunately, experimental evidence suggests that the applicability of this heuristics is limited, if one is interested in automatically finding complexity bounds, see Section 5 for further details. However, the standard approach for automatically proving termination via polynomial interpretations as stipulated by Contejean et al. 11] can be adapted. The description of this adaption is the topic of this section. We restrict the form of parametric interpretations that we consider.

Definition 10. $A$ (parametric) $\Delta$-linear interpretation is a polynomial context dependent interpretation $\mathcal{C}$ whose interpretation functions and parameter functions have the following form:

$$
\begin{aligned}
f_{\mathcal{C}}\left(\Delta, z_{1}, \ldots, z_{n}\right) & =\sum_{i=1}^{n} a_{(f, i)} z_{i}+\sum_{i=1}^{n} b_{(f, i)} z_{i} \Delta+c_{f} \Delta+d_{f} \\
f_{\mathcal{C}}^{i}(\Delta) & =\frac{\Delta}{a_{(f, i)}+b_{(f, i)} \Delta}
\end{aligned}
$$

where the occurring coefficients are supposed to be natural numbers. For a parametric $\Delta$-linear interpretation, $a_{(f, i)}, b_{(f, i)}, c_{f}$, and $d_{f}(f \in \mathcal{F}, 1 \leqslant i \leqslant n)$ are called coefficient variables.

Note that for any $\Delta$-linear interpretation, we have $a_{(f, i)}>0$ or $b_{(f, i)}>0(f \in \mathcal{F}$, $1 \leqslant i \leqslant n)$ : Any $\Delta$-linear interpretation is a polynomial context dependent interpretation by definition. And hence the parameter functions have to be $\Delta$ quotients, cf. Definition 7. Moreover the coefficients $a_{(f, i)}, b_{(f, i)}$ are used in the interpretation function and the parameter functions. This is necessary for the correctness of Lemma 12 below.

Example 11. Consider the TRS $\mathcal{R}_{1}$ from Example 1. The parametric interpretation and parameter functions have the form:

$$
\begin{array}{ll}
\mathrm{a}_{\mathcal{C}}[\Delta](z)=a z+b z \Delta+c \Delta+d & \mathrm{a}_{\mathcal{C}}^{1}(\Delta)=\frac{\Delta}{a+b \Delta} \\
\mathrm{b}_{\mathcal{C}}[\Delta](z)=e z+f z \Delta+g \Delta+h & \mathrm{~b}_{\mathcal{C}}^{1}(\Delta)=\frac{\Delta}{e+f \Delta} .
\end{array}
$$

The following lemma is a direct consequence of the definitions.

Lemma 12. Let $\mathcal{C}$ be an $\Delta$-linear interpretation. Then $\mathcal{C}$ is $\Delta$-monotone.

Due to Lemma 12 in order to prove termination of a given TRS $\mathcal{R}$, it suffices to find a $\Delta$-linear interpretation compatible with $\mathcal{R}$. This observation is reflected in the following definition. 
Definition 13. Let $\mathcal{R}$ be a TRS and let $\mathcal{C}$ be a parametric $\Delta$-linear interpretation. The compatibility constraints of $\mathcal{R}$ with respect to $\mathcal{C}$ are defined as

$$
\begin{aligned}
\mathrm{CC}(\mathcal{R}, \mathcal{C})= & \{[\alpha, \Delta](l)-[\alpha, \Delta](r)-\Delta \geqslant 0 \mid l \rightarrow r \in \mathcal{R}\} \cup \\
& \cup\left\{a_{(f, i)}+b_{(f, i)}-1 \geqslant 0 \mid f \in \mathcal{F}, 1 \leqslant i \leqslant \operatorname{ar}(f)\right\} .
\end{aligned}
$$

Here $\operatorname{ar}(f)$ denotes the arity of $f$ and $\alpha$ refers to a symbolic $\Delta$-assignment: Expressions of the form $[\alpha, \Delta](x)$ for $x \in \mathcal{V}$ remain unevaluated.

While the first half of $\operatorname{CC}(\mathcal{R}, \mathcal{C})$ represents compatibility with $\mathcal{R}$, the second set of constraints guarantees that the denominators of the occurring $\Delta$-quotients are different from 0 . Thus any solution to $\mathrm{CC}(\mathcal{R}, \mathcal{C})$, instantiating coefficients with natural numbers, represents a polynomial context dependent interpretation compatible with $\mathcal{R}$.

Example 14. Consider the (parametric) $\mathrm{CDA} \mathcal{C}$ from Example 11 and set $\Delta_{1}=$ $\mathrm{a}_{\mathcal{C}}^{1}(\Delta)$ and $\Delta_{2}=\mathrm{b}_{\mathcal{C}}^{1}(\Delta)$. Let $\alpha_{1}=\left[\alpha, \Delta_{2}\left[\Delta:=\Delta_{1}\right]\right](x)$ and let $\alpha_{2}=\left[\alpha, \Delta_{1}[\Delta:=\right.$ $\left.\left.\Delta_{2}\right]\right](x)$. Then the constraint $[\alpha, \Delta](\mathrm{a}(\mathrm{b}(x)))-[\alpha, \Delta](\mathrm{b}(\mathrm{a}(x)))-\Delta \geqslant 0$ becomes:

$$
\begin{gathered}
\left(a e \alpha_{1}+a f \alpha_{1} \Delta_{1}+a g \Delta_{1}+b e \alpha_{1} \Delta+b f \alpha_{1} \Delta_{1} \Delta+b g \Delta_{1} \Delta+(b h+c) \Delta+\right. \\
+a h+d)-\left(a e \alpha_{2}+b e \alpha_{2} \Delta_{2}+c e \Delta_{2}+a f \alpha_{2} \Delta+b f \alpha_{2} \Delta_{2} \Delta+c f \Delta_{2} \Delta+\right. \\
+(d f+g) \Delta+d e+h)-\Delta \geqslant 0 .
\end{gathered}
$$

For all constraints $(P \geqslant 0) \in \mathrm{CC}(\mathcal{R}, \mathcal{C}), P$ is an extended polynomial, cf. Lemma 8 It is easy to see how an extended polynomial (over $\left.\Delta, z_{1}, \ldots, z_{n}\right)$ is transferable into a (standard) polynomial (over $\Delta, z_{1}, \ldots, z_{n}$ ): Multiply (symbolically) with denominators of (nontrivial) $\Delta$-quotients till all (nontrivial) $\Delta$ quotients are eliminated. This simple procedure is denoted as A. Correctness and termination of the procedure follow trivially.

Definition 15. Let $\mathcal{R}$ be a TRS and let $\mathcal{C}$ be a parametric $\Delta$-linear interpretation. The polynomial compatibility constraints of $\mathcal{R}$ with respect to $\mathcal{C}$ are defined as follows: $\operatorname{PCC}(\mathcal{R}, \mathcal{C}):=\left\{P^{\prime} \geqslant 0 \mid P \geqslant 0 \in \mathrm{CC}(\mathcal{R}, \mathcal{C})\right.$ and $\left.P^{\prime}:=\mathrm{A}(P)\right\}$.

Example 16. Consider the constraint $P \geqslant 0$ depicted in Example 14 To apply the algorithm A we first have to symbolically multiply with the expression $a+b \Delta$ and later with $e+f \Delta$. The resulting constraint $P^{\prime} \geqslant 0$ (with the polynomial $P^{\prime}$ in the "variables" $\Delta, \alpha_{1}$, and $\alpha_{2}$ ) has the form:

$$
\begin{gathered}
\left(\left(b^{2} e f+b f^{2}\right) \alpha_{1} \Delta^{3}+\left(2 a b e f+a f^{2}+b^{2} e^{2}+b e f\right) \alpha_{1} \Delta^{2}\right. \\
\left.+\left(2 a b e^{2}+a^{2} e f+a e f\right) \alpha_{1} \Delta+\left(a^{2} e^{2}\right) \alpha_{1}\right) \\
-\left(\left(a b f^{2}+b^{2} f\right) \alpha_{2} \Delta^{3}+\left(a^{2} f^{2}+2 a b e f+a b f+b^{2} e\right) \alpha_{2} \Delta^{2}\right. \\
\left.+\left(2 a^{2} e f+a b e^{2}+a e b\right) \alpha_{2} \Delta+\left(a^{2} e^{2}\right) \alpha_{2}\right)+\left(\left(b^{2} f h-b d f^{2}-b f\right) \Delta^{3}\right. \\
+\left(2 a b f h+b^{2} e h+b d f-a d f^{2}-2 b d e f-b f h-b e-a f\right) \Delta^{2} \\
\left.+\left(a^{2} f h+2 a b e h+a d f+b d e-2 a d e f-a f h-b d e^{2}-b e h-a e\right)\right) \Delta \\
\left.+\left(a^{2} e h+a d e-a d e^{2}-a e h\right)\right) \geqslant 0 .
\end{gathered}
$$


We obtain $\operatorname{PCC}\left(\mathcal{R}_{1}, \mathcal{C}\right)=\left\{P^{\prime} \geqslant 0, a+b-1 \geqslant 0, e+f-1 \geqslant 0\right\}$, where the last two constraints reflect that all denominators of $\Delta$-quotients are non-zero.

Let $P \geqslant 0$ be a constraint in $\operatorname{PCC}(\mathcal{R}, \mathcal{C})$ such that $n$ distinct symbolic assignments $[\alpha, d](x)$ occur in $P(x \in \mathcal{V}, d$ a $\Delta$-quotient). (In Example 16 two symbolic assignments occur: $\alpha_{1}$ and $\alpha_{2}$.) Then $P$ is conceivable as a polynomial in $\mathbb{Z}\left[\Delta, z_{1}, \ldots, z_{n}\right]$. It remains to verify that (a suitable instance of) $P$ is positive, i.e., we have to prove that $P\left(\Delta, z_{1}, \ldots, z_{n}\right) \geqslant 0$ for any values $\Delta>0$, $z_{i} \geqslant 0$. This is achieved by testing for absolute positivity instead of positivity, compare [11.

A polynomial $P$ is absolutely positive if $P$ has non-negative coefficients only. A parametric polynomial $P$ is called absolutely positive if there exists an instance $P^{\prime}$ of $P$ such that $P^{\prime}$ is absolutely positive. Clearly any absolutely positive polynomial is positive. Thus for a given constraint $P \geqslant 0 \in \operatorname{PCC}(\mathcal{R}, \mathcal{C})$ it suffices to find instantiations of the coefficient variables such that all coefficients are natural numbers. This is achieved through the construction of suitable Diophantine inequalities over the coefficients.

Lemma 17. Let $\mathcal{R}$ be a TRS and let $\mathcal{C}$ denote a parametric $\Delta$-linear interpretation. If for all $P \geqslant 0 \in \operatorname{PCC}(\mathcal{R}, \mathcal{C}), P$ is absolutely positive then there exists an instantiation of $\mathcal{C}$ compatible with $\mathcal{R}$.

Proof. If $P$ is absolutely positive, there exist natural numbers that can be substituted to the coefficient variables in $P$ such that the resulting polynomial $P^{\prime}$ is absolutely positive and thus positive. By definition this implies that the constraints in $\operatorname{CC}(\mathcal{R}, \mathcal{V})$ are fulfilled. We define an instantiation $\mathcal{C}^{\prime}$ of $\mathcal{C}$ by applying the same substitution to the coefficient variables in $\mathcal{C}$. Then $\mathcal{C}^{\prime}$ is compatible with $\mathcal{R}$.

As an immediate consequence of Lemmata 12, 17, and Theorem 2 we obtain the following theorem.

Theorem 18. Let $\mathcal{R}$ be a TRS and let $\mathcal{C}$ denote a parametric $\Delta$-linear interpretation. Suppose for all $P \geqslant 0 \in \operatorname{PCC}(\mathcal{R}, \mathcal{C}), P$ is absolutely positive. Then $\mathcal{R}$ is terminating and property (1) holds for $D=\mathbb{R}^{+}$.

It is easy to see that the Diophantine inequalities induced by Example 16 cannot be solved, if the symbolic assignments $\alpha_{1}$ and $\alpha_{2}$ are treated as different variables. This motivates the next definition.

Definition 19. Given a TRS $\mathcal{R}$ and a $\Delta$-linear interpretation $\mathcal{C}$, the equality constraints of $\mathcal{R}$ with respect to $\mathcal{C}$ are defined as follows:

$$
\mathrm{EC}(\mathcal{R}, \mathcal{C})=\{(a+b \Delta)-(c+d \Delta)=0 \mid \text { Property }(*) \text { is fulfilled }\}
$$

(*) There exists $P \geqslant 0 \in \operatorname{PCC}(\mathcal{R}, \mathcal{C}), x \in \mathcal{V}$ such that $\left[\alpha, d_{1}\right](x)$ and $\left[\alpha, d_{2}\right](x)$ occur in $P$ and $d_{1}=\frac{\Delta}{a+b \Delta} \neq \frac{\Delta}{c+d \Delta}=d_{2}$. 
Example 20. Consider Example 16 . Property $(*)$ is applicable to the $\Delta$-quotients $d_{1}, d_{2}$ in the $\Delta$-assignments $\alpha_{1}=\left[\alpha, d_{1}\right]$ and $\alpha_{2}=\left[\alpha, d_{2}\right]$ as

$$
d_{1}=\frac{\Delta}{a e+(b e+f) \Delta} \neq \frac{\Delta}{a e+(a f+b) \Delta}=d_{2} .
$$

Thus the constraint $(a e+(b e+f) \Delta)-(a e+(a f+b) \Delta)=0$ occurs in $\operatorname{EC}\left(\mathcal{R}_{1}, \mathcal{C}\right)$. This is the only constraint in $\operatorname{EC}\left(\mathcal{R}_{1}, \mathcal{C}\right)$.

Let $P \geqslant 0 \in \operatorname{PCC}(\mathcal{R}, \mathcal{C})$, assume the equality constraints in $\operatorname{EC}(\mathcal{R}, \mathcal{C})$ are fulfilled and assume we want to test for absolute positivity of $P$. By assumption distinct symbolic assignments can be treated as equal, which may change the coefficients we need to consider in $P$. This is expressed by writing $P \geqslant 0 \in$ $\operatorname{PCC}(\mathcal{R}, \mathcal{C}) \cup \mathrm{EC}(\mathcal{R}, \mathcal{C})$. Furthermore, we call a parametric polynomial a zero polynomial if there exists an instance $P^{\prime}$ of $P$ such that $P^{\prime}=0$.

Corollary 21. Let $\mathcal{R}$ be a TRS and let $\mathcal{C}$ denote a parametric $\Delta$-linear interpretation. Suppose for all $P \geqslant 0(P=0) \in \operatorname{PCC}(\mathcal{R}, \mathcal{C}) \cup \mathrm{EC}(\mathcal{R}, \mathcal{C}), P$ is absolutely positive ( $P$ is a zero polynomial). Then $\mathcal{R}$ is terminating and property (1) holds for $D=\mathbb{R}^{+}$.

Corollary 21 opens the way to efficiently search for CDAs: Finding a $\Delta$ monotone and compatible CDA $\mathcal{C}$ amounts to solving the Diophantine constraints in $\operatorname{PCC}(\mathcal{R}, \mathcal{C}) \cup \mathrm{EC}(\mathcal{R}, \mathcal{C})$. It is well-known that solvability of Diophantine constraints is undecidable [12. However, there is an easy remedy for this: we restrict the domain of the coefficient variables to a finite one.

Example 22. Consider the TRS $\mathcal{R}_{1}$ from Example 1 and the $\Delta$-linear interpretation $\mathcal{C}$ from Example 11. Applying the above described algorithm, the following Diophantine (in)equalities need to be solved.

$$
\begin{array}{ll}
b^{2} e f+b f^{2}-a b f^{2}-b^{2} f \geqslant 0 & a b e^{2}+a^{2} e f+a e f-2 a^{2} e f-a e b \geqslant 0 \\
b^{2} f h-b d f^{2}-b f \geqslant 0 & a^{2} e h+a d e-a d e^{2}-a e h \geqslant 0 \\
a+b-1 \geqslant 0 & e+f-1 \geqslant 0 \\
b e+f-a f-b=0 & a f^{2}+b^{2} e^{2}+b e f-a^{2} f^{2}-a b f-b^{2} e \geqslant 0 \\
2 a b f h+b^{2} e h+b d f-a d f^{2}-2 b d e f-b f h-b e-a f \geqslant 0 & \\
a^{2} f h+2 a b e h+a d f+b d e-2 a d e f-a f h-b d e^{2}-b e h-a e \geqslant 0 .
\end{array}
$$

Here the constraints $a+b-1 \geqslant 0, e+f-1 \geqslant 0$ guarantee that the denominators of occurring $\Delta$-quotients are positive, and the equality $b e+f-a f-b=0$ expresses the equality constraint in $\operatorname{EC}\left(\mathcal{R}_{1}, \mathcal{C}\right)$. Our below discussed implementations of the algorithm presented in this section find the following satisfying assignments for the coefficient variables fully automatically:

$$
a=b=e=h=1 \quad c=d=f=g=0 .
$$




\section{Derivational Complexities Induced by Polynomial Context Dependent Interpretations}

In this section we show that the derivational complexity induced by $\Delta$-linear interpretations is exponential and that this bound is tight. Furthermore, we introduce a restricted subclass of $\Delta$-linear interpretations that induces (tight) quadratic derivational complexity.

Recall the TRS $\mathcal{R}_{1}$ considered in Examplen 1 . This TRS belongs to a family of TRSs $\mathcal{R}_{k}$ for $k>0: \mathrm{a}(\mathrm{b}(x)) \rightarrow \mathrm{b}^{k}(\mathrm{a}(x))$ and it is not difficult to see that for $k \geqslant 2$ the derivational complexity of $\mathcal{R}_{k}$ is exponential. In [7] $\Delta$-linear interpretations $\mathcal{C}_{k}$ were introduced such that

$$
\inf _{\Delta>0} \frac{[\alpha, \Delta]_{\mathcal{C}_{k}}(t)}{\Delta}=\mathrm{dl}_{\mathcal{R}_{k}}(t),
$$

holds for any ground term. I.e., for all $k>0$ there exist $\Delta$-linear interpretations that optimally bound the derivational complexities of $\mathcal{R}_{k}$. This triggers the question whether we can find such context dependent interpretations automatically. The next example answers this question affirmatively, for $k=21$

Example 23. Consider the TRSs $\mathcal{R}_{2}: \mathrm{a}(\mathrm{b}(x)) \rightarrow \mathrm{b}(\mathrm{b}(\mathrm{a}(x))) \mathrm{H}^{2}$ To find a $\Delta$-linear interpretation, we employ the same parametric interpretation $\mathcal{C}$, as in Example 11 and build the set of constraints $\mathrm{CC}\left(\mathcal{R}_{2}, \mathcal{C}\right)$ and consecutively the polynomial compatibility constraints $\operatorname{PCC}\left(\mathcal{R}_{2}, \mathcal{C}\right)$ together with the equality constraints $\mathrm{EC}\left(\mathcal{R}_{2}, \mathcal{C}\right)$. We only state the (automatically) obtained interpretation and parameter functions:

$$
\begin{array}{ll}
\mathrm{a}_{\mathcal{C}}[\Delta](z)=(2+2 \Delta) z & \mathrm{a}_{\mathcal{C}}^{1}(\Delta)=\frac{1}{2+2 \Delta} \\
\mathrm{b}_{\mathcal{C}}[\Delta](z)=z+1 & \mathrm{~b}_{\mathcal{C}}^{1}(\Delta)=\Delta .
\end{array}
$$

As a consequence of Example 23 we see the existence of TRSs, compatible with $\Delta$-linear interpretations, whose derivational complexity function is exponential. Moreover, we have the following lemma.

Lemma 24. Let $\mathcal{C}$ denote a $\Delta$-linear interpretation and let $K$ denote the maximal coefficient occurring in $\mathcal{C}$. Further let $t$ be a ground term, $\alpha$ a $\Delta$-assignment and $\Delta>0$. Then $[\alpha, \Delta](t) \leqslant(K+2)^{|t|}(\Delta+1)$.

Proof. Straightforward induction on $t$.

Theorem 25. Let $\mathcal{R}$ be a TRS and let $\mathcal{C}$ denote a $\Delta$-linear interpretation compatible with $\mathcal{R}$. Then $\mathcal{R}$ is terminating and $\operatorname{dc}_{\mathcal{R}}(n)=2^{\mathcal{O}(n)}$. Moreover there exists a TRS $\mathcal{R}$ such that $\mathrm{dc}_{\mathcal{R}}(n)=2^{\Omega(n)}$.

\footnotetext{
${ }^{1}$ The answer remains positive for $k=3$. Detailed experimental evidence and additional information on the considered constraints are available at http:// cl-informatik.uibk.ac.at/ aschnabl/experiments/cdi/

${ }_{2}^{2}$ This is Example 2.50 in Steinbach and Khler's collection [13].
} 
Proof. The proof of the upper bound follows the pattern of the proof of Theorem 29 below. To show that this upper bound is tight, we consider the TRS $\mathcal{R}_{2}$ from Example 23. It is easy to see that $\mathrm{dc}_{\mathcal{R}_{2}}(n)=2^{\Omega(n)}$ holds.

In order to establish a termination method that induces polynomial derivational complexity, we restrict the class of $\Delta$-linear interpretations.

Definition 26. $A \Delta$-restricted interpretation is a $\Delta$-linear interpretation. In addition we require that for the interpretation functions and parameter functions

$$
\begin{aligned}
f_{\mathcal{C}}\left(\Delta, z_{1}, \ldots, z_{n}\right) & =\sum_{i=1}^{n} a_{(f, i)} z_{i}+\sum_{i=1}^{n} b_{(f, i)} z_{i} \Delta+c_{f} \Delta+d_{f} \\
f_{\mathcal{C}}^{i}(\Delta) & =\frac{\Delta}{a_{(f, i)}+b_{(f, i)} \Delta},
\end{aligned}
$$

we have $a_{(f, i)} \in\{0,1\}$ for all $1 \leqslant i \leqslant n$.

Example 27. Consider the TRS $\mathcal{R}_{1}$ from Example 1. The assignment of coefficient variables as defined in Example 22 induces a $\Delta$-restricted interpretation.

Lemma 28. Let $\mathcal{C}$ denote a $\Delta$-restricted interpretation with coefficients $a_{(f, i)}$, $b_{(f, i)}, c_{f}, d_{f}(f \in \mathcal{F}, 1 \leqslant i \leqslant \operatorname{ar}(f))$ and we set

$$
\begin{aligned}
M & :=\max \left(\left\{c_{f}, d_{f} \mid f \in \mathcal{F}\right\} \cup\{1\}\right) \\
N & :=\max \left(\left\{b_{(f, i)} \mid f \in \mathcal{F}, 1 \leqslant i \leqslant \operatorname{ar}(f)\right\} \cup\{1\}\right) .
\end{aligned}
$$

Further let $t$ be a ground term, $\alpha$ a $\Delta$-assignment and let $\Delta>0$. Then $[\alpha, \Delta](t) \leqslant$ $M\left(|t|+N|t|^{2} \Delta\right)$.

Proof. We proceed by induction on $t$. As $t \in \mathcal{T}(\mathcal{F})$, the evaluation is independent of the assignment. Hence we write $[\Delta](t)$ instead of $[\alpha, \Delta](t)$. If $t=f \in \mathcal{F}$, then

$$
[\Delta](t)=c_{f} \Delta+d_{f} \leqslant M(\Delta+1) \leqslant M\left(|t|+N|t|^{2} \Delta\right) .
$$

If on the other hand $t=f\left(t_{1}, \ldots, t_{n}\right)$, then

$$
\begin{aligned}
{[\Delta](t) } & =\sum_{i}\left(a_{f_{i}}+b_{f_{i}} \Delta\right)\left[f_{\mathcal{C}}^{i}(\Delta)\right]\left(t_{i}\right)+c_{f} \Delta+d_{f} \\
& \leqslant \sum_{i}\left(a_{f_{i}}+b_{f_{i}} \Delta\right)\left(M\left(\left|t_{i}\right|+N\left|t_{i}\right|^{2} \frac{\Delta}{a_{f_{i}}+b_{f_{i}} \Delta}\right)+c_{f} \Delta+d_{f}\right. \\
& =\sum_{i}\left(\left(a_{f_{i}}+b_{f_{i}} \Delta\right) M\left|t_{i}\right|+M N\left|t_{i}\right|^{2} \Delta\right)+c_{f} \Delta+d_{f} \\
& \leqslant \sum_{i}\left((1+N \Delta) M\left|t_{i}\right|+M N\left|t_{i}\right|^{2} \Delta\right)+M(\Delta+1) \\
& \leqslant \sum_{i}\left|t_{i}\right|((1+N \Delta) M+M N(|t|-1) \Delta)+M(\Delta+1) \\
& =(|t|-1)((1+N \Delta) M+M N(|t|-1) \Delta)+M(\Delta+1) \\
& =M\left((|t|-1)(1+N \Delta)+N(|t|-1)^{2} \Delta+(\Delta+1)\right) \\
& \leqslant M\left(|t|+N|t|^{2} \Delta\right) .
\end{aligned}
$$


In line (3) we employ the induction hypothesis, in (6) we use $\left|t_{i}\right| \leqslant|t|-1$ and for (9) a simple calculation reveals: $(|t|-1)(1+N \Delta)+N \Delta(|t|-1)^{2}+(\Delta+1)=$ $|t|+N|t|^{2} \Delta+\Delta-N|t| \Delta \leqslant|t|+N|t|^{2} \Delta$.

Theorem 29. Let $\mathcal{R}$ be a TRS and let $\mathcal{C}$ denote a $\Delta$-restricted interpretation compatible with $\mathcal{R}$. Then $\mathcal{R}$ is terminating and $\mathrm{dc}_{\mathcal{R}}(n)=\mathcal{O}\left(n^{2}\right)$. Moreover there exists a TRS $\mathcal{R}$ such that $\mathrm{dc}_{\mathcal{R}}(n)=\Omega\left(n^{2}\right)$.

Proof. By Theorem $2 \mathcal{R}$ is terminating and by Lemma 28 , there exists $K \in \mathbb{N}$, such that for any ground term $t:[\Delta](t) \leqslant K\left(|t|+K|t|^{2} \Delta\right) \leqslant K^{2}|t|^{2}(\Delta+1)$ and hence

$$
\mathrm{dl}_{\mathcal{R}}(t) \leqslant \inf _{\Delta>0} \frac{[\Delta](t)}{\Delta} \leqslant \inf _{\Delta>0} \frac{K^{2}|t|^{2}(\Delta+1)}{\Delta}=K^{2}|t|^{2} .
$$

We obtain $\operatorname{dl}_{\mathcal{R}}(t)=\mathcal{O}\left(|t|^{2}\right)$ for any $t \in \mathcal{T}(\mathcal{F}, \mathcal{V})$ and thus $\operatorname{dc}_{\mathcal{R}}(n)=\mathcal{O}\left(n^{2}\right)$. The tightness of the bound follows by Example 1 .

By definition the constant employed in Theorem 29 depends only on the employed interpretation functions. Moreover this dependence is linear. In concluding this section, we want to stress that $\Delta$-restricted interpretation are even strong enough to handle non-simple terminating TRSs.

Example 30 ([7]). Consider the TRS $\mathcal{R}$ with the one rule $\mathrm{a}(\mathrm{a}(x)) \rightarrow \mathrm{a}(\mathrm{b}(\mathrm{a}(x)))$. By applying the algorithm described in Section 3 , we find the below given $\Delta$ restricted interpretation $\mathcal{C}$ automatically:

$$
\mathrm{a}_{\mathcal{C}}[\Delta](z)=2 z \Delta+2 \quad \mathrm{~b}_{\mathcal{C}}[\Delta](z)=z \Delta \quad \mathrm{a}_{\mathcal{C}}^{1}(\Delta)=\frac{1}{2} \quad \mathrm{~b}_{\mathcal{C}}^{1}(\Delta)=1 .
$$

By Theorem 18, $\mathcal{C}$ is compatible with $\mathcal{R}$. Hence Theorem 29 implies that the derivational complexity of $\mathcal{R}$ is (at most) quadratic.

\section{Experimental Results}

In this section we describe the programs $c \mathrm{cdi}_{1}, \mathrm{cdi}_{2}$, and $\mathrm{cdi}_{3}$. These programs provide search procedures for context dependent interpretations. The program $\mathrm{cdi}_{1}$ implements the heuristics of Hofbauer in [7, mentioned in Section 3 above. On the other hand, programs $\mathrm{cdi}_{2}$ and $\mathrm{cdi}_{3}$ implement the algorithm presented in Section 3 and incorporate constraint solvers for Diophantine (in)equalities. The program $\mathrm{cdi}_{1}$ searches for $\Delta$-linear interpretations, while $\mathrm{cdi}_{2}$ and $\mathrm{cdi}_{3}$ can search for $\Delta$-linear and $\Delta$-restricted interpretations. We summarise further differences below:

$\mathrm{cdi}_{1}$ Firstly, the program searches for a polynomial interpretation compatible with a TRS $\mathcal{R}$. This interpretation is then lifted to a polynomial context dependent interpretation $\mathcal{C}$ as follows: Coefficients of the form $k+1$ are replaced by $k+\Delta$. Finally Mathematics ${ }^{3}$ is invoked to verify that the resulting CDA $\mathcal{C}$ is $\Delta$-monotone and compatible with $\mathcal{R}$.

\footnotetext{
3 http://www . wolfram.com/products/mathematica/.
} 
$\mathrm{Cdi}_{2}$ This programs employs a constraint propagation procedure to solve the Diophantine constraints in $\operatorname{PCC}(\mathcal{R}, \mathcal{C}) \cup \mathrm{EC}(\mathcal{R}, \mathcal{C})$. Essentially the implementation follows the technique suggested in [11].

$\mathrm{cdi}_{3}$ The Diophantine (in)equalities in $\operatorname{PCC}(\mathcal{R}, \mathcal{C}) \cup \mathrm{EC}(\mathcal{R}, \mathcal{C})$ are translated into propositional logic and suitable assignments are found by employing a SAT solver, in our case MiniSat ${ }^{4}$ The implementation follows ideas presented in [14] and employs the plogic library of $\mathrm{T}_{\mathrm{T}} \mathrm{T}_{2}{ }^{5}$

The implementation of the transformation steps as described in Section 3 , is the same for $\mathrm{cdi}_{2}$ and $\mathrm{cdi}_{3}$. The programs $\mathrm{cdi}_{1}, \mathrm{cdi}_{2}$, and $c \mathrm{cdi}_{3}$ are written in OCamb (and parts of $\mathrm{cdi}_{1}$ in $\mathrm{C}$ ). All three programs are fairly small: cdi ${ }_{1}$ consists of about 2000 lines of code, while $\mathrm{cdi}_{2}$ and $\mathrm{cdi}_{3}$ use roughly 3000 lines of code each.In Table 1 we summarise the comparison between the different programs $\mathrm{cdi}_{1}, \mathrm{cdi}_{2}$, and $\mathrm{cdi}_{3}$. The numbers in the third line of the table refer to the number of bits maximally used in cdi $i_{3}$ to encode coefficients. Correspondingly for $\mathrm{cdi}_{2}$ we used 32 as strict bound on the coefficients. We are interested in automatically verifying the complexity of terminating TRSs. Consequentially, as testbed we employ those 957 TRSs from the version 4.0 of the Termination Problem Data Base (TPDB for short) that can be shown terminating with at least one of the tools that participated in the termination competition 2007 7 The presented tests were performed single-threaded on a $2.40 \mathrm{GHz}$ Intel@ Core $^{\mathrm{TM}} 2$ Duo with $2 \mathrm{~GB}$ of memory. For each system we used a timeout of 60 seconds, the times in the tables are given in milliseconds.

Table 1. 957 terminating TRSs

\begin{tabular}{l|c|cc|cccc|ccccc} 
& $\mathrm{cdi}_{1}$ & \multicolumn{4}{|c}{$\mathrm{cdi}_{2}$} & \multicolumn{1}{c}{$\mathrm{cdi}_{3}$} \\
& $\Delta$-linear & $\Delta$-restr. $\Delta$-linear & \multicolumn{4}{c}{$\Delta$-restricted } & \multicolumn{4}{c}{$\Delta$-linear } \\
& & & & 2 & 3 & 4 & 5 & 2 & 3 & 4 & 5 \\
\hline \hline \# success & 19 & 61 & 62 & 83 & 86 & 86 & 86 & 82 & 82 & 82 & 83 \\
average time & - & 3132 & 3595 & 3652 & 4041 & 4008 & 3986 & 5496 & 4981 & 5010 & 5527 \\
\# timeout & - & 276 & 782 & 144 & 189 & 222 & 238 & 525 & 687 & 751 & 797
\end{tabular}

Observe that the heuristic proposed in [7 is not suitable as an automatic procedure. (We have not indicated the time spent by $\mathrm{cdi}_{1}$ as the timing is incomparable to the stand-alone approach of $\mathrm{cdi}_{2}$ or $\mathrm{cdi}_{3}$.) With respect to the comparison between $\mathrm{cdi}_{2}$ and $\mathrm{cdi}_{3}$, the latter outperforms the former, if at least 2 bits are used. Perhaps surprisingly the performance of $\mathrm{cdi}_{2}$ and $\mathrm{cdi}_{3}$ on $\Delta$ restricted and $\Delta$-linear is almost identical. This can be explained by the strong impact of larger bounds for the coefficients $a_{(f, i)}(f \in \mathcal{F}, 1 \leqslant i \leqslant \operatorname{ar}(f))$ in the

\footnotetext{
4 http://minisat.se/

5 http://colo6-c703.uibk.ac.at/ttt2/

6 http://www.caml.inria.fr/,

7 These 957 systems and full experimental evidence can be found at http:// cl-informatik.uibk.ac.at/ aschnabl/experiments/cdi/
} 
complexity of the issuing Diophantine (in)equalities. However, for both programs $\mathrm{cdi}_{2}$ and $\mathrm{cdi}_{3}$, the stronger technique gains one crucial system: Example 23 .

Table 2 relates existing methods that induce polynomial derivational complexities of TRSs to $\mathrm{cdi}_{3}$. SL refers to strongly linear interpretations, i.e., only interpretation functions of the form $f_{\mathcal{A}}\left(x_{1}, \ldots, x_{n}\right)=\sum_{i} x_{i}+c, c \in \mathbb{N}$ are allowed. Clearly compatibility with strongly linear interpretations induces linear derivational complexity. Secondly, TTTbox refers to the implementation of the matchbound technique as in [15: Linear TRSs are tested for match-boundedness, nonlinear, but non-duplicating TRSs are tested for match-raise-boundedness. This technique again implies linear derivational complexity. (Employing [16] (as in [5]) one sees that any match-raise bounded TRS has linear derivational complexity. Then the claim follows from Lemma 8 in 15.) Note that the restriction to non-duplicating TRS is harmless, as any duplicating TRS induces at least exponential derivational complexity. No further termination methods that induce at most polynomial derivational complexities for TRSs have previously been known. In particular related work on implicit complexity (for example [17|18|19|20|21]) does not provide methods that induce polynomial derivational complexities, even if sometimes the derivation length can be bounded polynomially, if the set of start terms is suitably restricted. Finally $\mathrm{cdi}^{+}$denotes our standard strategy: First, we search for a strongly linear interpretation. If such an interpretation cannot be found, then a $\Delta$-restricted interpretation is sought (with 5 bits as bound).

Table 2. Termination Methods as Complexity Analysers

\begin{tabular}{l|c|c|c|c} 
& $\mathrm{SL}$ & $\mathrm{T}_{\mathrm{T}}$ Tbox & $\mathrm{cdi}_{3}-\Delta$-restricted & $\mathrm{cdi}^{+}-\Delta$-restricted \\
\hline \hline \# success & 41 & 125 & 86 & 87 \\
average time & 20 & 577 & 3986 & 3010 \\
\# timeout & 0 & 225 & 238 & 237
\end{tabular}

Some comments on the results reported in Table 2. By definition the set of TRSs compatible with a strongly linear interpretation is a (strict) subset of those treatable with $\mathrm{cdi}^{+}$. On the other hand the comparison between TTTbox and cdi ${ }^{+}$ (or $\mathrm{cdi}_{3}$ ) may appear not very favourable for our approach. However, $\mathrm{cdi}^{+}$(and $\mathrm{cdi}_{3}$ ) can handle TRSs that cannot be handled by TTTbox. More precisely with respect to $\Delta$-restricted interpretations $\mathrm{cdi}^{+}$(and $\mathrm{cdi}_{3}$ ) can handle 38 (37) TRSs that cannot be handled with TTTbox. For instance the following example can only be handled with $\mathrm{cdi}^{+}$(and $\mathrm{cdi}_{3}$ ).

Example 31. Consider the following rewrite system $\mathcal{R}_{+,-}$(This is Example 2.11 in Steinbach and Khler's collection [13.)

$$
\begin{aligned}
& 0+y \rightarrow y \quad 0-y \rightarrow x \quad \mathrm{~s}(x)-\mathrm{s}(y) \rightarrow x-y \\
& \mathrm{~s}(x)+y \rightarrow \mathrm{s}(x+y) \quad x-0 \rightarrow x
\end{aligned}
$$


It is easy to see that $\mathcal{R}_{+,-}$is compatible with the following (automatically generated) $\Delta$-restricted interpretation $\mathcal{C}$.

$$
\begin{aligned}
-_{\mathcal{C}}[\Delta](x, y) & =x+y+3 y \Delta+2 \Delta & 0_{\mathcal{C}}[\Delta] & =0 \\
+_{\mathcal{C}}[\Delta](x, y) & =x+y+x \Delta+\Delta & \mathrm{s}_{\mathcal{C}}[\Delta](x) & =x+2,
\end{aligned}
$$

with parameter functions: $-{ }_{\mathcal{C}}^{1}(\Delta)=+{ }_{\mathcal{C}}^{2}(\Delta)=\mathrm{s}_{\mathcal{C}}^{1}(\Delta=) \Delta,-{ }_{\mathcal{C}}^{2}(\Delta)=\frac{\Delta}{1+3 \Delta}$, and $+{ }_{\mathcal{C}}^{1}(\Delta)=\frac{\Delta}{1+\Delta}$. Due to Theorem 29 we conclude quadratic derivational complexity, while the standard polynomial interpretation would only allow to conclude an exponential upper bound. Note that the deduced quadratic derivational complexity provides an optimal upper bound.

Another issue is the high average yes time (and the higher number of timeouts) of $\mathrm{cdi}_{3}$ and $\mathrm{cdi}^{+}$in relation to existing techniques. Although a closer look reveals that the total times spent by $\mathrm{T}_{\mathrm{T}} \mathrm{Tb}$ box and $\mathrm{cdi}^{+}\left(\right.$or $\mathrm{cdi}_{3}$ ) is relatively equal, an improvement of the efficiency of the introduced tools seems worthwhile.

Remark 32. Note that $\mathrm{cdi}^{+}$in conjunction with $\mathrm{T}_{\mathrm{T}} \mathrm{T}$ box can automatically verify that 163 TRSs in the testbed are of at most quadratic derivational complexity. Put differently more than $10 \%$ of all 1381 TRSs (and more than a third of the 445 non-duplicating TRSs) in version 4.0 of the TPDB are of quadratic derivational complexity.

\section{Conclusion}

In this paper we have presented two subclasses of context dependent interpretations, and established tight upper bounds on the induced derivational complexities. More precisely, we have delineated two subclasses: $\Delta$-linear and $\Delta$-restricted context dependent interpretations that induce exponential and quadratic derivational complexity, respectively. Further, we introduced an algorithm for mechanically proving termination of rewrite systems with context dependent interpretations. As a consequence we established a technique to automatically verify quadratic derivational complexity of TRSs. Finally, we reported on different implementations of this algorithm and presented numerical data to compare these implementations with existing methods that allow to automatically verify polynomial derivational complexity of TRSs.

We believe the here presented approach can be extended further. A starting point for future work would be to decide whether it is possible to define additional subclasses of context dependent interpretations inducing polynomial derivational complexities that grow faster than quadratic. One possible approach is to drop the restriction to integer coefficients and thus generalise the notion of polynomial context dependent interpretations. By Tarski's quantifier elimination method, such an extension turns the undecidable positivity problem for Diophantine (in)equalities into a decidable problem. Further research will clarify the impact of this extension. A crucial problem in practical considerations is the known ineffectivity of quantfier elimination, see for example [22]. 


\section{References}

1. Hofbauer, D., Lautemann, C.: Termination proofs and the length of derivations. In: Proc. 3rd RTA. Volume 355 of LNCS. (1989) 167-177

2. Hofbauer, D.: Termination proofs by multiset path orderings imply primitive recursive derivation lengths. TCS 105 (1992) 129-140

3. Weiermann, A.: Termination proofs for term rewriting systems with lexicographic path orderings imply multiply recursive derivation lengths. TCS 139 (1995) 355362

4. Moser, G.: Derivational complexity of Knuth Bendix orders revisited. In: Proc. 13th LPAR. Volume 4246 of LNCS. (2006) 75-89

5. Geser, A., Hofbauer, D., Waldmann, J., Zantema, H.: On tree automata that certify termination of left-linear term rewriting systems. IC 205 (2007) 512-534

6. Hirokawa, N., Moser, G.: Automated complexity analysis based on the dependency pair method. In: Proc. 4th IJCAR, Springer (2008) accepted for publication.

7. Hofbauer, D.: Termination proofs by context-dependent interpretations. In: Proc. 12th RTA. Volume 2051 of LNCS. (2001) 108-121

8. Baader, F., Nipkow, T.: Term Rewriting and All That. Cambridge University Press (1998)

9. Terese: Term Rewriting Systems. Volume 55 of Cambridge Tracts in Theoretical Computer Science. Cambridge University Press (2003)

10. Schnabl, A.: Context Dependent Interpretations. Master's thesis, Universität Innsbruck (2007) Available at http://cl-informatik.uibk.ac.at/ aschnabl/,

11. Contejean, E., Marché, C., Tomás, A.P., Urbain, X.: Mechanically proving termination using polynomial interpretations. JAR 34 (2005) 325-363

12. Matiyasevich, Y.: Enumerable sets are diophantine. Soviet Mathematics (Dokladi) 11 (1970) 354-357

13. Steinbach, J., Kühler, U.: Check your ordering - termination proofs and open problems. Technical Report SEKI-Report SR-90-25, University of Kaiserslautern (1990)

14. Fuhs, C., Giesl, J., Middeldorp, A., Schneider-Kamp, P., Thiemann, R., Zankl, H.: SAT solving for termination analysis with polynomial interpretations. In: Proc. 10th SAT. Volume 4501 of LNCS. (2007) 340-354

15. Korp, M., Middeldorp, A.: Proving termination of rewrite systems using bounds. In: Proc. 18th RTA. Volume 4533 of LNCS. (2007) 273-287

16. Hofbauer, D., Waldmann, J.: Deleting string rewriting systems preserve regularity. TCS 327 (2004) 301-317

17. Bonfante, G., Cichon, A., Marion, J.Y., Touzet, H.: Algorithms with polynomial interpretation termination proof. JFP 11 (2001) 33-53

18. Marion, J.Y.: Analysing the implicit complexity of programs. IC 183 (2003) 2-18

19. Bonfante, G., Marion, J.Y., Moyen, J.Y.: Quasi-intepretations and small space bounds. In: Proc. 16th RTA. Volume 3467 of LNCS. (2005) 150-164

20. Marion, J.Y., Péchoux, R.: Resource analysis by sup-interpretation. In: Proc. 8th FLOPS. Volume 3945 of LNCS. (2006) 163-176

21. Avanzini, M., Moser, G.: Complexity analysis by rewriting. In: Proc. 9th FLOPS. Volume 4989 of LNCS. (2008) 130-146

22. Caviness, B., Johnson, J., eds.: Quantifier Elimination and Cylindrical Algebraic Decomposition. Springer (2004) 